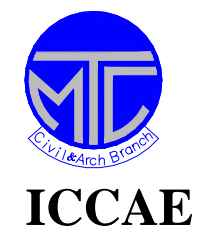

Military Technical College

Kobry Elkobbah, Cairo, Egypt 6hㅡ International Conference

On Civil \& Architecture

Engineering

\title{
COMBINED GENETIC AND SEQUENTIAL QUADRATIC PROGRAMMING ALGORITHMS FOR VIBRATION- BASED STRUCTURAL HEALTH MONITORING
}

\author{
S.Y. MAHFOUZ * and M. S. AMIN *
}

\begin{abstract}
Structural Health Monitoring (SHM) of a structure is a requirement desirable for many engineering structures in both civil and aerospace applications. Early warning of impeding disasters, if not identified on time, damage may have serious consequences, both safety related and economic. However, the complexity of large structures and the difficulty in accessing them makes the use of traditional Non-Destructive Evaluation (NDE), such as visual inspection and instrument evaluation methods, impractical. An effective alternative is the use of methods that depend on Vibration-Based Damage Identification (VBDI). These methods are based on measurement of changes in the modal characteristic of the structure, that is, its frequencies and mode shapes, to identify changes in its physical properties. Therefore, measurement and monitoring of vibration characteristics should theoretically permit the detection of both the location and severity of damage.

In the present paper, the output error method of damage identification has been used to assess the presence and severity of damage in steel structures. The identification problem is formulated as optimization problem to find parameters describing the presence and location of damaged elements in a structure by minimization of discrepancy of the modal response predicted by FE analysis from the test data (mixed analytical-experimental approach). A typical portal frame has been chosen for experimental measurements. The modal parameters were measured through modal testing. Two approaches are introduced to solve the identification problem. The first approach deals with a discrete problem that is solved by Genetic Algorithm (GA). The second treats a mixed discrete-continuous problem that is solved by a combination of GA and Sequential Quadratic Programming (SQP), in which the GA is used to produce a high quality starting guess for the (SQP) method that is applied to accurately identify damage. It is found that the first approach was able to identify damage in case of moderate and sever simulated damage, while the second approach is capable to identify damage in case of light damage.
\end{abstract}

* Ph.D, Civil Engineering Department, Military Technical College, Egyptian Armed Forces, Egypt. 
Proceedings of the 6th ICCAE Conf. 16 -18 May, 2006

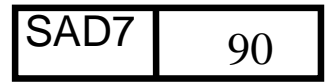

\section{KEYWORDS}

Vibration-based damage detection, structural health monitoring, Optimization, Sequential Quadratic Programming, Genetic Algorithm.

\section{INTRODUCTION}

Early detection of damage to civil engineering structures has assumed a special importance because of the aging infrastructure, increased demand, complexity and size of some of the modern structures, and lack of long-term experience with innovative materials and structural shapes that may be incorporated in a structure.

Damage may be detected by visual inspection or instrumental evaluation. These methods require that all portions of the structure are accessible. This may be impractical, particularly when the structure is complex and/or large in size. Certain type of damage, for example, internal delamination and fibre fracture in a composite, and fracture of prestressing strands in a prestressed concrete girder cannot be detected by visual inspection. Several NDE techniques have been developed to detect damage that may not be visible to the naked eye. Application of such methods requires a-prior knowledge of the possible damage sites and access to such sites. Also, the results of instrumental evaluation are often inconclusive or difficult to evaluate.

A great deal of research has been carried out in the past decade or so on the development of analytical techniques of vibration-based damage detection. Early work in the damage identification algorithms for SHM mainly depended on the variation in the modal frequencies to detect damage, Cawley \& Adams [1], named output error approach, used the first order perturbation of the basic eigenvalue equation to obtain sensitivities necessary to locate the damage in a structure using natural frequencies. The location of the damage was assumed to be where the theoretically determined ratio of changes in any two frequencies was equal to the experimentally measured value. West [2] presented a method for the systematic use of mode shape information for the location of structural damage without the use of a prior FEM. An alternative to use mode shapes to obtain information about sources of vibration changes is to utilize mode shape derivatives, such as curvature. This method was proposed by Pandey et al. [3]. Stubbs and Kim [4] introduced an algorithm that relied on the evaluation of a parameter called Damage Index to identify damage in a beam type structure. The most widely used damage detection algorithms are the optimal matrix update methods [5,6]. They rely on the solution of a nonlinear optimization problem formulated on the basis of the structure's equations of motion, properties of the nominal model, and the measured dynamic characteristics. Doebling et al [7] provide an extensive bibliography related to this subject. Farrar et al [8] have reviewed the literature on vibration testing and damage detection in bridges. More recently, Amin [9] presented an integrated SHM system based on the use of frequencies and mode shapes for damage detection in a 3D frame. The damage was modelled on an element-by-element basis as changes in elemental stiffness matrices, which then contribute to variation in the terms of the structural stiffness matrix. 
In the output error approach, changes are made in analytical model to match the experimental response, where in the and the equation error approach the model parameters are adjusted to obtain a match between the left and right hand sides of the response equation. The mathematical optimization techniques depends a great deal on the availability of a baseline FEM that has the ability to predict the measured dynamic response of the undamaged structure. However, even when such a model is available, the methods by themselves may not perform well when used to detect damage in complex structures, often providing ambiguous results. In the present study, two mathematical approaches were used to implement the output error approach of damage identification to assess the presence and severity of different levels of simulated damage in portal steel frame. The first approach deals with a discrete problem that is solved by Genetic Algorithm (GA). The second treats a mixed discrete-continuous problem that is solved by a combination of GA and Sequential Quadratic Programming (SQP), in which the GA is used to produce a high quality starting guess for the (SQP) method that is applied to accurately identify damage. It is found that the first approach was able to identify damage in case of moderate and sever simulated damage, while the second approach is capable to identify damage in case of light damage.

\section{IDENTIFICATION PROBLEM FORMULATION}

Vibration-based damage detection algorithms use the basic eigenvalue equation represented in terms of the matrices $\mathbf{K}$ and $\mathbf{M}$. For healthy structure the equation is formulated as follows to determine the i-th eigenvalue $\omega_{i}^{2}$, and the corresponding eigenmode $\xi_{\mathrm{i}}$.

$$
\left(\mathbf{K}-\omega_{i}^{2} \mathbf{M}\right) \xi_{i}=0
$$

Matrices $\mathbf{K}$ and $\mathbf{M}$ are to be adjusted to minimize the differences between the experimentally measured eigenvalues and values obtained from the analytical (e.g., finite element) model. As a variation in the system matrices results in changed frequency response, the damage assessment problem, formulated as an inverse problem, is to relate these differences to changes in specific elements of the system matrices. In order to describe the influence of the presence and extent of damage on the matrices $\mathbf{K}$ and $\mathbf{M}$, the optimization variables $\mathbf{x}$ are to be introduced such as sectional properties of individual structural elements (the crosssectional area, moments of inertia) or material parameters (Young's modulus, etc.).

Two basic approaches can be suggested for the description of the presence, location and the extent of structural damage by optimization variables $x$. In the first one, an individual variable xi can describe the extent of possible damage at i-th location, e.g. in the i-th finite element. This formulation leads to a continuous optimization problem, can easily describe the presence of multiple damage but the number of variables can be large when a large scale finite element model is used. Alternatively, the vector of variables can be considered as a set of $\mathrm{L}$ couples $x_{1}^{1}, x_{2}^{1}, x_{1}^{2}, x_{2}^{2}, \ldots, x_{1}^{L}, x_{2}^{L}$ where $x_{1}^{j}$ is a number of a damaged element and $x_{2}^{j}$ describes the extent of damage occurring in it, $\mathrm{j}=1, \ldots, \mathrm{L}$ and $\mathrm{L}$ is the assumed maximum number of damaged elements. Such approach leads to a considerably smaller number of variables but presents a discrete or mixed discrete-continuous optimization problem. In the present study both approaches has been adopted. 
Using the output error approach, the damage identification problem can be formulated to find the values of optimization variables $\mathrm{x}$ by minimizing the differences between the frequencies $\omega_{i}^{M}$ measured in the course of laboratory experiment or operation and the frequencies $\omega_{i}^{A}(\mathbf{x})$ obtained by the finite element analysis:

$$
\text { Minimize }\left[\omega_{i}^{M}-\omega_{i}^{A}(x)\right]^{2}, \mathrm{i}=1, \ldots, \mathrm{F}
$$

where $\mathrm{F}$ is total number of modes of vibration used for the identification.

The formulated problem is a multi-criterion one but it can be transformed to a more traditional optimization problem by formulating a single criterion. The linear combination of individual differences equation (2) is a most typically used one, the optimization problem can then be reformulated in the following form:

$$
\text { Minimize } \sum_{i=1}^{F} w_{i}\left\{\left[\omega_{i}^{M}-\omega_{i}^{A}(\mathbf{x})\right] / \omega_{i}^{M}\right\}^{2}
$$

where the weights $w_{i}$ describe the relative importance of the match between frequencies of the i-th mode. This optimization problem has the following characteristic features: (i) the objective function is an implicit function of parameters $\boldsymbol{x}$, (ii) to calculate values of this function for the specific set of parameters $\boldsymbol{x}$ means to use the finite element simulation of the structure under consideration, which can involve a large amount of computer time, (iii) function values present some level of noise, i.e. can only be estimated with finite accuracy. The direct implementation of any of conventional nonlinear mathematical programming techniques would involve too large amount of computer time and, moreover, the convergence of a method cannot be guaranteed due to the presence of numerically induced noise in the objective function values and its derivatives.

\section{EXPERIMENTAL PROCEDURE}

The test structure used for the investigation was a steel portal frame clamped at the base of both columns as shown in Figure 1. All parts of the frame have the same $800 \mathrm{~mm} \times 400 \mathrm{~mm}$ rectangular hollow section of $4 \mathrm{~mm}$ thickness. The first ten natural frequencies were measured on undamaged frame and also when three stages of progressive damage (classified as mild, medium and severe) were applied at the location close to the top joint. In all cases, the damage was applied by removing the material symmetrically relative to the beam's neutral axis thus reducing the cross section area to $64 \%, 54 \%$ and $35 \%$ of the original value for the undamaged structure.

For the experimental data acquisition a standard technique of modal structural testing has been used. Natural frequencies were measured by the impulse technique because of its speed and ease of execution. The oscillations in the structure have been excited with an instrumented hammer with a build-in force transducer Bruel \& Kjaer (B\&K) type 8200. The acceleration of frame was measured by using a $14 \mathrm{~g}$ accelerometer (B\&K type 4369) so the 
weight of that comparing to the weight of the frame was negligible so that it do not affect the total mass of the frame. The signals from hammer and accelerometer are amplified by B\&K charge amplifiers type 2635.

The excitation and response signals have been measured and processed using the dual channel spectral analyzer B\&K type 2032. It transforms the two sampled time functions into frequency spectra by a Fast Fourier Transformation (FFT) and subsequently computes the ratio of these functions yielding the Frequency Response Function (FRF). For example two measured FRF at the same response point for undamaged frame(solid curve) and damaged frame (dashed curve) are shown in Fig. 2. The difference between natural frequencies for damaged and undamaged frame can easily be seen. As expected, the natural frequency for damaged structure is lower than for undamaged one. The adequate number of the accelerometer positions along the perimeter of the frame have been established to ensure that no resonance is overlooked. To obtain sufficient resolution in low frequencies the measurement has been repeated in different frequency bands (25, 50, 100, 200 and $400 \mathrm{~Hz}$.). Some additional measurements were executed to detect and eliminate out of plane mode shapes and frequencies. The results are shown in Table 1.

A finite element model of 56 plane beam elements was created using the ANSYS 5.4. The built in optimization Algorithm ANSYS was used to update the FE model parameters using experimental results on ten first natural frequencies for undamaged frame by minimizing the difference between experimental and analytical results. Four parameters have then initially been considered as optimization variables: the Young's modulus and the density of the material, area of cross section, and the moment of inertia of small artificially introduced elements at the base of both columns. Variation in the last parameter was intended to cover the uncertainty of boundary conditions (clamped columns) and had the most profound effect on validation. In addition, the effect in changes in the FE mesh was studied and found to be insignificant. The results of FE model updating are presented in Table 1.

\section{THEORITICAL BACKGROUND OF (GA) \& (SQP)}

The solution of the optimization problem using measured modal parameters can be implemented using different numerical optimization techniques. Some of these techniques are derivative-based algorithms such as Quadratic Programming (QP), Sequential Quadratic Programming (SQP) and Linear Matrix Inequality (LMI). Others are probabilistic-based techniques such as Genetic Algorithms (GA) (Friswell et al [10]) and Simulated Annealing. A better understanding of the mathematical bases of these algorithms is crucial in the selection of the technique to be used in the proposed mathematical methodology. It is undesirable to have a false prediction of damage location and severity due to poor performance of the optimization technique used. Each technique has its own advantages and drawbacks. In the following subsections, the mathematical background of used optimization techniques will be briefly discussed. 


\section{Genetic algorithm}

Genetic algorithm (GA) is an optimization strategy in which points in the design space are analogous to organisms involved in a process of natural selection. The term genetic is used because, along with the expected design representation, GA employs a code representation of design attributes that is analogous to a chromosome (see, Holland [11] and [12]). This code is commonly a character string, with each character position being analogous to a gene, and each character assigned to a position being analogous to an allele. Organisms are generated and tested in generations, with offspring designs arising from parent designs. The creation of new designs for a new generation occurs with a process that is analogous to biological reproduction. Genetic crossover allows offspring designs to retain traits from parent designs, and infrequent mutations possibly yield radically improved designs. The testing of new designs is done with merit function, usually tailored to take the coded representation as input. In a given generation, designs with a higher merit are given a higher probability of creating offspring, and perhaps surviving themselves into the next generation.

Optimization occurs, therefore, through a process of natural selection. Designs in a given generation group in pairs (i.e., mate), with the better designs having a higher probability of pairing. These parent designs produce offspring by genetic crossover. In single point crossover, a point along the coded representations (the chromosomes) is chosen at random, and the segments of the code after the point are swapped. After that random mutations are performed on individual alleles within the chromosomes by changing the values. These operations yield two new codes which represent two new designs that possess traits from both parents. The process then iterates. After many generations, the best design is achieved, because the merit function is more likely to allow better designs to produce offspring. Generally, the GA is judged to be successful if it evolves a population of highly fit individuals. Among many authors, Goldberg [13], Davis [14] and Michalewicz [15] may be consulted for more details regarding the various aspects of genetic algorithms.

\section{Sequential Quadratic Programming}

In constrained optimization, the general aim is to transform the problem into an easier subproblem that can be solved and used as the basis of an iterative process. Efficient methods have been developed that depend on the solution of Kuhn-Tucker (KT) equations. The solution of the KT equations forms the basis of many non-linear optimization algorithms. These algorithms attempt to compute directly the Lagrange multipliers. However methods that employ constrained quasi-Newton updating procedure guarantee super-linear convergence by accumulating second order information regarding the KT equations. These methods are commonly referred to as Sequential Quadratic Programming (SQP), since a quadratic programming sub-problem is solved at each major iteration. SQP is also considered as the state of the art in the non-linear optimization field. This method allows the user to closely minimize Newton's method for constrained optimization just as is done for unconstrained optimization. At each major iteration, an approximation is made of the Hessian of the Lagrangian function using a quasi-Newton updating method. This is then used to generate a QP sub-problem whose solution is used to form a search direction for a line search procedure. For more details , Haftka et al. [16] may be consulted. 


\section{IMPLEMENTATION OF THE PROPOSED APPROACHES}

In order to simplify the problem, at first, The GA is utilized to find the location and severity of the damage at only one damaged point. Here, the vector of variables of the optimization problem equation (3), is presented as a set of $\mathrm{L}$ couples $x_{1}^{1}, x_{2}^{1}, x_{1}^{2}, x_{2}^{2}, \ldots, x_{1}^{L}, x_{2}^{L}$ where $\mathrm{L}$ is the assumed maximum number of damaged elements. In each couple $x_{1}^{j}$ is a number of a damaged element describing the location of damage (a discrete variable) and $x_{2}^{j}$ was proposed as a number between 1 and 128 describing the extent of damage occurring at a corresponding $j$-th location. Such approach leads to a considerably smaller number of optimization variables, so the number of possible damage locations can be easily increased, and this is the most important benefit of this approach.

The first trial, five possible places for the damage were nominated. Because of the symmetry condition, only three optimization variables were defined in the optimization problem, the first place is at the top next to the corner, the second is located at the third is at the base. Both the location and size of the damage were successfully detected. In order to determine the number of modes necessary to be used for reliable detection of damage, the number of modes was incremented one by one, the results are presented in Table 2 . As could be expected, for a mild damage at least first four natural frequencies were needed to detect the damage but for the medium and severe damage the first three and two modes respectively were sufficient.

After considering the first results, it was assumed that the damage could happen at a greater number of possible locations. Thus eleven possible locations were considered: five additional possible places of damage on a rafter and three other places on a column in addition to the three places mentioned earlier in the first trial. There was no restriction on the number of damaged elements. The first six natural frequencies were used to detect the damage. The location and size of the damage were successfully detected again applying the GA only, the results are presented in Table 3.

The cross-sectional areas of eight elements of the FE model were considered as optimization variables. Lower and upper bounds of these cross-sectional areas were taken as 1 and 128 respectively, where 100 (or near) represents an undamaged element. The discretization of design variables was defined by increments by 1 thus resulting in the overall string length of 56 for all of the eight design variables. The following parameters of the genetic algorithm have been used: size of the population 60, proportion of the elite of 0.4 , the probabilities of crossover and mutation have been taken as 0.6 and 0.01 respectively. The computations were carried out for three, five and eight possible damaged locations and in all cases damage was successfully detected. But it was found that for a greater number of optimization variables the run time of program to achieve accurate results has been excessively long. The results for damage detection with eight possible damage places (optimization variables) are shown in Table 4. It has been found that the GA is capable to identify damage in case of moderate and sever simulated damage.

Next, in order to deal with more accurate optimization problem, the mixed discretecontinuous optimization problem is proposed, in which a two step approach is employed so that the GA is applied to identify an initial guess of the vector of design variables that is needed for the start of running the SQP technique. In case of mild damage, the change in the 
natural frequencies is considerably small, accordingly the two step approach has been applied. The number of possible damage places was assumed first 15 and, in the second attempt, 31 and, accordingly, the upper bounds of the variables $x_{1}^{j}$ were taken as 16 and 32, respectively. The lower bounds of these variables were taken as 1 . The lower and upper bounds of cross-sectional areas, $x_{2}^{j}$, were taken as 1 and 128 respectively. The assumed maximum number of damaged element, L, was assumed to be one, two and three in three successive damage detection runs. In all cases the damage was successfully detected, the results are shown in Tables 5 and 6.

\section{CONCLUDING REMARKS}

In SHM using VBDI techniques, the solution of the optimization problem is the last step in the damage identification algorithms. The physical implication of finding a local minimum solution is that a false prediction of the damage site and extent has been obtained. Getting false prediction due to poor performance of the optimization technique used would spoil the whole identification system. The presence of a large number of design variables in the objective function is another factor that affects the performance of the optimization technique evolving directly from the size of the physical model. In other words when identifying damage in large complex structures, if all the structural elements represented in the FEM are considered as candidates in the optimization problem, the number of design variables would be large.

The implementation of GA for vibration-based damage identification technique would be successful only if a small number of possible design variables is introduced for any level of damage severity. However, in practice it is difficult to identify the possible damaged sites in advance and if so, a considerably large number of possible site should be addressed. Consequently, the use of the GA only in such cases would be time consuming and may lead to false prediction of damage severity.

The use of the proposed two step approach proves to insure the localization of damage site for any level of damage ranging from mild to sever and accurately identify the degree of severity. This can be attributed to the combination of the two optimization techniques used, in which in the first step the probabilistic based GA technique is used to identify the initial guess design variables vector. In the second step the vector of initial guess is used as a start for the implementation of the derivative based SQP technique. This methodology is meant to simplify the role of the SQP so that it hits the correct solution which physically represents the damage identification. 


\section{REFERENCES}

[1] Cawley, P. and Adams, R. D., The Location of Defects in Structures From Measurements of Natural Frequencies, Journal of Strain Analysis 14(2), 1979, pp. 49-57.

[2] West, W. M., Illustration of the Use of Modal Assurance Criterion to Detect Structural Changes in an Orbiter Test Specimen, in Proceedings of the air Force Conference on aircraft Structural Integrity, 1984, pp.1-6.

[3] Pandey, A. K., et al, Damage Detection from Changes in Curvature Mode Shapes, Journal of Sound and Vibration 145(2), 1991, pp.321-332.

[4] Stubbs, N. and Kim, J. T., Field Verification of a Non-Destructive Damage Localization and Severity Estimation Algorithm, Texas A\&M University Report prepared for New Mexico State University, 1994.

[5] Hossiotis, S. and Jeong, G.D., Identification of Stiffness Reductions Using Natural Frequencies, ASCE, Journal of Engineering Mechanics, Vol. 121, No. 10, October 1995.

[6] Li, W. L., A New Method for FEM Structural Dynamic Model Updating and Joint Identification, 18th International Modal Analysis Conference, IMAC, San Antonio, Texas, Feb. 3-6, 2000, pp. 454-460.

[7] Doebling, S. W., Farrar, C. R., Prim, M. B., and Shevitz, D. W., Damage Identification and Health Monitoring of Structural and Mechanical Systems From Changes in Their Vibration Characteristics: A Literature Review, Los Alamos National Laboratory Report LA-13070-MS., 1996.

[8] Farrar, C.R, Baker, W.E., Bell, T.M., Cone, K.M., Darling, T.W., Duffey, T.A., Eklund, A., Migliori, A. (1994). "Dynamic Characterization and Damage Detection in the I-40 Bridge Over the Rio Grande,” Los Alamos National Laboratory, Los Alamos, New Mexico, Report No. LA 12767-MS.

[9] Amin, M. S., "An Integrated Vibration-Based Structural Health Monitoring System", Ph.D. thesis submitted to Carleton University, Ottawa, Canada, 2002.

[10] Friswell, M. I., Penny, J. E. T and Gravey, S. D. "A combined genetic and eigensensetivity algorithm for the location of damage in structures", Computers and Structures Vol. 69, December 1998.

[11] Holland, J.H., “Adaptation in Natural and Artificial Systems”, University of Michigan, Ann Arbor, 1975.

[12] Holland, J.H., "Adaptation in Natural and Artificial Systems. An Introductory Analysis with Applications to Biology, Control and Artificial Intelligence”, MIT press, 1992.

[13] Goldberg, D.E., "Genetic Algorithms in Search, Optimization and Machine Learning”. Addison-Wesley Publishing Company, Inc., 1989.

[14] Davis, L., "Handbook of Genetic Algorithms", Van Nostrand Reinhold, New York,1991.

[15] Michalewicz, Z., "Genetic Algorithm + Data Structures = Evolution Programs”, 3rd ed., Springer-Verlag, New York, 1996.

[16] Haftka, R. T., Gurdal, Z. and Kamat, M. P., "Elements of Structural Optimization", Kluwer Academic Publishers, London,1993. 
Table 1. Experimental and analytical values of natural frequencies

\begin{tabular}{|c|c|c|c|c|c|c|c|c|c|}
\hline \multirow{4}{*}{$\begin{array}{l}\text { Mode } \\
\text { num- } \\
\text { ber }\end{array}$} & \multicolumn{5}{|c|}{ Undamaged frame } & \multirow{2}{*}{\multicolumn{4}{|c|}{$\begin{array}{c}\text { Damaged frame } \\
\text { Experimental }\end{array}$}} \\
\hline & \multirow{3}{*}{$\begin{array}{c}\text { Experi- } \\
\text { ment } \\
\text { Freq., } \\
\text { Hz }\end{array}$} & \multicolumn{4}{|c|}{ Analytical (FEM) } & & & & \\
\hline & & \multicolumn{2}{|c|}{ Before updating } & \multicolumn{2}{|c|}{ After updating } & \multicolumn{4}{|c|}{$\begin{array}{ll}\text { Mild damage } & \text { Medium damage }\end{array}$} \\
\hline & & $\begin{array}{c}\text { Freq., } \\
\mathrm{Hz}\end{array}$ & $\begin{array}{l}\text { Difference, } \\
\%\end{array}$ & $\begin{array}{c}\text { Freq., } \\
\mathrm{Hz}\end{array}$ & $\begin{array}{l}\text { Difference, } \\
\%\end{array}$ & $\begin{array}{c}\text { Freq., } \\
\mathrm{Hz}\end{array}$ & $\begin{array}{c}\text { Change } \\
\%\end{array}$ & $\begin{array}{c}\text { Freq., } \\
\mathrm{Hz}\end{array}$ & $\begin{array}{c}\text { Change } \\
\%\end{array}$ \\
\hline 1 & 12.59 & 15.41 & 22.39 & 12.61 & 0.12 & 12.63 & 0.25 & 12.59 & 0.00 \\
\hline 2 & 18.47 & 19.59 & 6.06 & 18.31 & -0.87 & 18.34 & -0.68 & 18.13 & -1.86 \\
\hline 3 & 44.13 & 45.34 & 2.75 & 43.65 & -1.09 & 44.13 & 0.00 & 44.13 & 0.00 \\
\hline 4 & 76.38 & 76.50 & 0.16 & 75.47 & -1.19 & 74.38 & -2.62 & 72.13 & -5.57 \\
\hline 5 & 128.5 & 135.0 & 5.09 & 128.1 & -0.33 & 128.5 & 0.00 & 128.5 & 0.00 \\
\hline 6 & 140.8 & 163.7 & 16.30 & 141.0 & 0.16 & 140.0 & -0.53 & 139.0 & -1.24 \\
\hline 7 & 173.8 & 198.9 & 14.50 & 175.0 & 0.73 & 173.8 & 0.00 & 173.5 & -0.14 \\
\hline 8 & 223.5 & 237.0 & 6.05 & 225.2 & 0.76 & 220.0 & -1.57 & 216.0 & -3.36 \\
\hline 9 & 306.5 & 313.7 & 2.34 & 308.4 & 0.60 & 306.0 & -0.16 & 305.5 & -0.33 \\
\hline 10 & 361.0 & 367.3 & 1.75 & 364.7 & 1.02 & 354.0 & -1.94 & 348.0 & -3.60 \\
\hline
\end{tabular}


Table 2. Damage detection and the number of modes used (three possible locations)

\begin{tabular}{|c|c|c|c|c|c|c|c|c|c|c|c|c|}
\hline \multirow{2}{*}{$\begin{array}{l}\text { Type of } \\
\text { actual } \\
\text { damage }\end{array}$} & \multicolumn{2}{|c|}{$\begin{array}{l}\text { Damage location } \\
\text { and extent }\end{array}$} & \multicolumn{10}{|c|}{$\begin{array}{l}\text { Number of frequencies used for damage identification and corresponding percentage of remaining area } \\
\text { of cross-section found }\end{array}$} \\
\hline & Joint & $\begin{array}{c}\text { Area } \\
(\%)\end{array}$ & 1 & 2 & 3 & 4 & 5 & 6 & 7 & 8 & 9 & 10 \\
\hline \multirow{3}{*}{ Mild } & 1 & 100 & 81 & 105 & 105 & 105 & 105 & 105 & 103 & 90 & 89 & 95 \\
\hline & 2 & 64 & 77 & 99 & 99 & 67 & 67 & 66 & 66 & 63 & 63 & 59 \\
\hline & 3 & 100 & 102 & 102 & 104 & 103 & 103 & 102 & 100 & 104 & 105 & 104 \\
\hline \multirow{3}{*}{ Medium } & 1 & 100 & 86 & 70 & 105 & 105 & 105 & 105 & 95 & 89 & 86 & 98 \\
\hline & 2 & 54 & 26 & 98 & 53 & 50 & 50 & 50 & 50 & 49 & 49 & 47 \\
\hline & 3 & 100 & 93 & 103 & 95 & 96 & 97 & 97 & 96 & 97 & 101 & 95 \\
\hline \multirow{3}{*}{ Severe } & 1 & 100 & 86 & 105 & 105 & 105 & 90 & 88 & 89 & 104 & 105 & 103 \\
\hline & 2 & 35 & 26 & 33 & 33 & 32 & 33 & 33 & 33 & 32 & 32 & 32 \\
\hline & 3 & 100 & 93 & 92 & 93 & 95 & 105 & 105 & 105 & 104 & 105 & 103 \\
\hline
\end{tabular}

Table 3. Damage detection using the first six natural frequencies (eleven possible locations)

\begin{tabular}{|c|c|c|c|c|c|c|c|c|c|c|c|c|}
\hline \multirow{2}{*}{$\begin{array}{l}\text { Type of actual } \\
\text { damage }\end{array}$} & \multicolumn{12}{|c|}{ Damage location and extent } \\
\hline & Element No. & 1 & 2 & 3 & 4 & 5 & 6 & 7 & 8 & 9 & 10 & 11 \\
\hline \multirow[b]{2}{*}{ Mild } & Exact area (\%) & 100 & 64 & 100 & 100 & 100 & 100 & 100 & 100 & 100 & 100 & 100 \\
\hline & Detected area & 105 & 65 & 105 & 105 & 93 & 101 & 105 & 105 & 105 & 98 & 101 \\
\hline \multirow[b]{2}{*}{ Medium } & Exact area (\%) & 100 & 54 & 100 & 100 & 100 & 100 & 100 & 100 & 100 & 100 & 100 \\
\hline & Detected area & 105 & 50 & 100 & 105 & 96 & 105 & 105 & 105 & 105 & 95 & 101 \\
\hline \multirow[b]{2}{*}{ Severe } & Exact area (\%) & 100 & 35 & 100 & 100 & 100 & 100 & 100 & 100 & 100 & 100 & 100 \\
\hline & Detected area & 100 & 32 & 102 & 105 & 96 & 98 & 105 & 105 & 105 & 93 & 95 \\
\hline
\end{tabular}

Table 4. Damage detection using GA (eight possible locations) 
Proceedings of the 6th ICCAE Conf. 16 -18 May, 2006

\begin{tabular}{|l|l|}
\hline SAD7 & 100 \\
\hline
\end{tabular}

\begin{tabular}{|c|c|c|c|c|c|c|c|c|}
\hline \multicolumn{10}{|c|}{ Damage location and extent } \\
\hline Element No. & 1 & 2 & 3 & 4 & 5 & 6 & 7 & 8 \\
\hline Exact area (\%) & 100 & 64 & 100 & 100 & 100 & 100 & 100 & 100 \\
\hline Detected area & 86 & 68 & 96 & 100 & 84 & 95 & 89 & 104 \\
\hline
\end{tabular}

Table 5. Damage detection using GA (15 possible damage locations)

\begin{tabular}{|c|c|c|c|c|c|c|c|c|}
\hline \multicolumn{8}{|c|}{ Damage location and extent } \\
\hline \multicolumn{2}{|c|}{ Element No. } & 1 & 2 & 3 & 4 & 5 & 6 & $7-15$ \\
\hline \multicolumn{2}{|c|}{ Exact area (\%) } & 100 & 64 & 100 & 100 & 100 & 100 & 100 \\
\hline \multirow{2}{*}{$\begin{array}{c}\text { The assumed } \\
\text { maximum number } \\
\text { of damage }(L)\end{array}$} & $L=1$ & 100 & 67 & 100 & 100 & 100 & 100 & 100 \\
\cline { 2 - 10 }$y$ & $L=2$ & 100 & 67 & 100 & 100 & 100 & 100 & 100 \\
\cline { 2 - 10 }$y$
\end{tabular}

Table 6. Damage detection using GA (31 possible damage locations)

\begin{tabular}{|c|c|c|c|c|c|c|c|c|c|c|c|}
\hline \multicolumn{10}{|c|}{ Damage location and extent } \\
\hline \multicolumn{2}{|c|}{ Element No. } & 1 & 2 & 3 & $4-8$ & 9 & 10 & 11 & $12-14$ & 15 & $16-31$ \\
\hline \multicolumn{2}{|c|}{ Exact area (\%) } & 100 & 64 & 100 & 100 & 100 & 100 & 100 & 100 & 100 & 100 \\
\hline \multirow{2}{*}{$\begin{array}{c}\text { The assumed } \\
\text { maximum number } \\
\text { of damage }(L)\end{array}$} & $L=1$ & 100 & 67 & 100 & 100 & 100 & 100 & 100 & 100 & 100 & 100 \\
\cline { 2 - 52 } & $L=2$ & 58 & 100 & 100 & 100 & 103 & 100 & 100 & 100 & 100 & 100 \\
\hline
\end{tabular}


Proceedings of the 6th ICCAE Conf. 16 -18 May, 2006

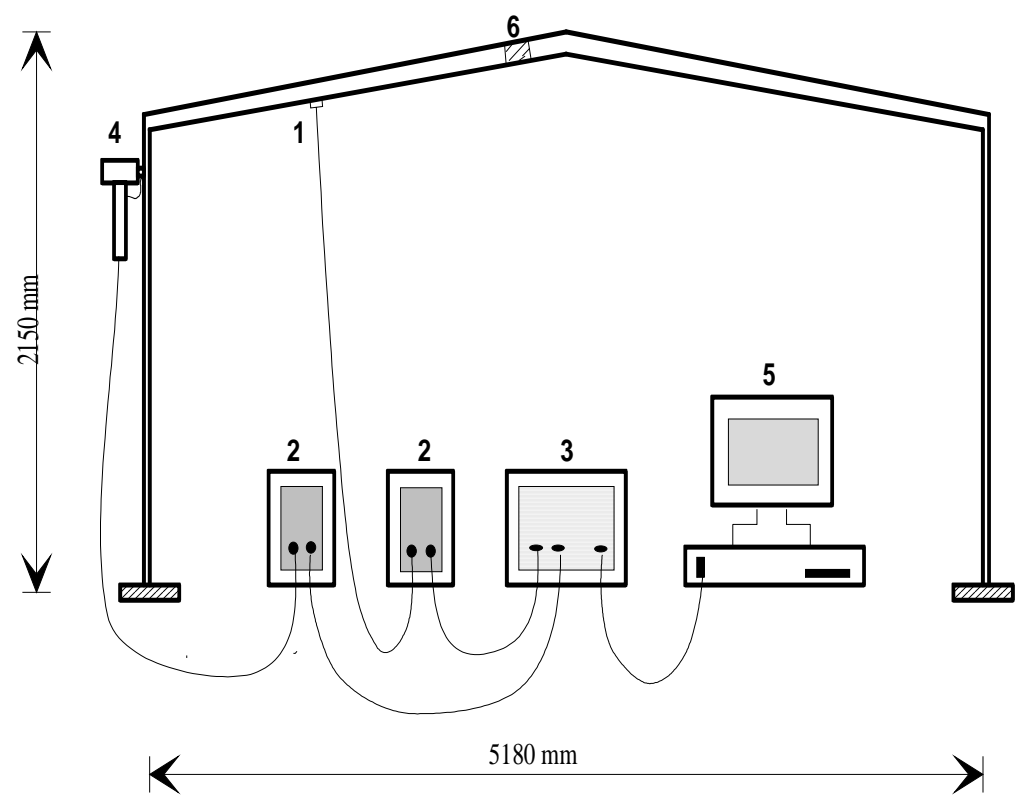

1) Accelerometer

2) Charge amplifier

3) Dual channel analyser 4) Instrumented hammer

5) Personal computer

6) Damage position

Fig. 1. The portal frame and instrumentation set up

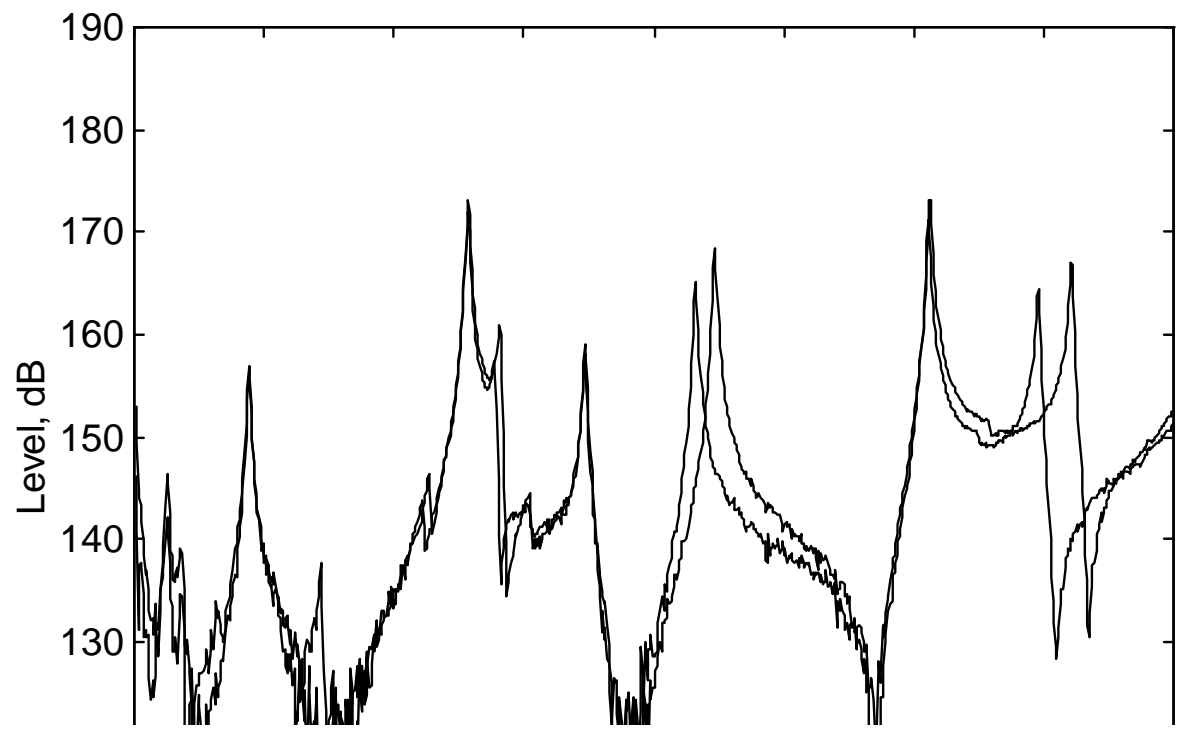

Fig. 2. Plot of frequency response functions for undamaged (solid curves) and damaged frame (dashed curves) 